\title{
Use of aVR and Jastrzębski algorithms in the classification of wide complex tachycardia in dogs - a preliminary study
}

\author{
Marcin Michałek ${ }^{1}$, Piotr Frydrychowski ${ }^{2}$, Jakub Adamowicz ${ }^{3}$, \\ Agnieszka Sławuta ${ }^{4}$, Urszula Pasławska ${ }^{1}$, Agnieszka Noszczyk-Nowak ${ }^{1}$ \\ ${ }^{1}$ Department of Internal Medicine and Clinic of Diseases of Horses, Dogs and Cats, Faculty of Veterinary Medicine, \\ ${ }^{2}$ Final-year student of Faculty of Veterinary Medicine, \\ Wrocław University of Environmental and Life Sciences, 50-366 Wrocław, Poland \\ ${ }^{3}$ Department of Clinical Nursing, Faculty of Health Sciences, \\ Wroclaw Medical University, 51-618 Wrocław, Poland \\ ${ }^{4}$ Department of Cardiology, Kłodzko County Hospital, 57-300 Kłodzko, Poland \\ m.michalek@outlook.com
}

Received: April 29, $2018 \quad$ Accepted: August 24, 2018

\begin{abstract}
Introduction: Ventricular rhythm disturbances are a common pathology in human and veterinary medicine. In humans, the algorithmic approach is used to differentiate wide QRS complex tachycardia. The most commonly used are the aVR and Brugada algorithms as well as the ventricular tachycardia (VT) score developed by Jastrzębski and coworkers. In veterinary medicine, no such algorithms are available and the only parameter used to describe VT abnormalities is the duration of the QRS complexes. The aim of this analysis was determining whether human medicine algorithms for VT are applicable in veterinary medicine to differentiate wide QRS complex tachycardia in dogs. Material and Methods: A retrospective analysis was performed on 11 dogs of both sexes and various breeds and age diagnosed with VT. The diagnosis was based on ambulatory ECG, further established based on the reaction to lidocaine or adenosine or an invasive electrophysiological study. Results: Of the 11 tracings passed through the aVR algorithm, 10 met the VT criteria. The most common criterion was the Vi/Vt ratio (8 out of 11 tracings). Based on the VT score, seven out of eight dogs had a high probability of VT. Conclusion: Retrospective analysis of ECGs by aVR and VT score indicates that the applied algorithms may be useful in differentiating wide QRS complex tachycardia as a quick, easy, and non-invasive alternative to cardiac electrophysiology.
\end{abstract}

Keywords: dog, ventricular tachycardia, wide-complex tachycardia, VT score, electrocardiography.

\section{Introduction}

Ventricular rhythm disturbances are a common pathology in human and veterinary medicine. Regardless of the aetiology, a re-entrant arrhythmia circuit is the cause of the electrophysiological substrate of most clinically significant arrhythmias. The re-entry mechanism contains a central block area, slow conduction zones, and a unidirectional block (2). All these components are necessary to produce and perpetuate the arrhythmia and may be of anatomical or functional origin, while the unidirectional block is particularly common in damaged heart areas.
A functional origin may cause the arrhythmia to be elusive in certain patients, and difficult or even impossible to induce during electrophysiological study in laboratory setting. Ventricular arrhythmia may be the result of enhanced automaticity and triggered by activity, although these mechanisms are clinically of limited significance. In humans and animals, single premature ventricular complexes may be present in healthy hearts, but their intensity (number per $24 \mathrm{~h}$ and the variety of ventricular morphology) is usually low. Complexes or clusters of ventricular beats, which are pairs or shorter or longer runs of ventricular tachycardia (VT), usually indicate the presence of 
a substantial underlying pathology, even if the morphological assessment (i.e. echocardiography) does not reveal large structural changes (10).

It is widely accepted that ventricular rhythm disturbances occur below the His bundle, including arrhythmia originating from the working myocardium and bundle branches as well as the fascicles of the interventricular conduction system. Their main features include the prolongation of the QRS complex (which may be different in human and various animal species) and distorted morphology when compared with the atrial activation. The presence of atrioventricular dissociation indicates the ventricular origin of any given arrhythmia (1). All the above-mentioned features can be misleading in specific clinical settings, so differentiation between ventricular and supraventricular origins of the arrhythmia is challenging.

VT can be monomorphic (if the QRS complexes are of the same shape) and polymorphic (different shapes of QRS complexes). Additionally, a duration below $30 \mathrm{~s}$ indicates non-sustained VT, while a duration of more than $30 \mathrm{~s}$ implies sustained VT. As mentioned above, ventricular QRS complexes are wide and distorted, although aberrantly conducted atrial arrhythmia and atrio-ventricular antidromic re-entrant tachycardia present the same feature, and their differentiation requires additional testing $(3,11)$. The interventricular conduction aberrancy may affect different levels of the His-Purkinje system, including the right and left bundle branches. A left anterior and posterior hemi block may be present due to the fact that the left bundle branch is usually divided into two fascicles. In addition, multifascicular, focal and diffuse interventricular blocks can be recognised. Each conduction abnormality has its own electrocardiographic characteristics with specific shapes of the QRS complex. In humans, the morphology can be additionally modified by preexisting changes of the QRS complex, for example related to myocardial necrosis. The presence of all the possible ECG changes associated with interventricular conduction abnormalities makes the differential diagnosis of the condition difficult $(3,11)$.

In the case of sustained wide QRS complex tachycardia, some pharmacological and nonpharmacological challenges may be helpful in the differentiation of its origin. One of these is the intravenous administration of adenosine. The substance temporarily negatively influences atrio-ventricular conduction, which enables tachycardia differentiation using the atrioventricular node. The Valsalva maneuver, which temporarily increases the vagal tone, is a common and easy non-pharmacological challenge, while other more sophisticated and demanding challenges require electrophysiological study (EPS). The use of the last approach enables a precise assessment of the arrhythmia substrate, the recognition of additional or pathological conduction pathways and diagnostic or curative heart pacing. Important limiting factors for further application of invasive EPS include the relatively high cost of the procedure and the need for sophisticated equipment and specialised $\operatorname{staff}(8)$.

In human medicine, algorithmic approaches are used to differentiate wide QRS complex tachycardia. The most commonly used are the aVR and Brugada algorithms as well as the VT score developed by Jastrzębski et al. $(1,6,7,12)$. In veterinary medicine, no such algorithms are available, and the only parameter used for VT description is the duration of the QRS complexes. It is widely accepted that a duration of the QRS complex above $50 \mathrm{~ms}$ or $60 \mathrm{~ms}$ in large animals indicates a ventricular origin of the tachycardia $(3,11)$. As previously mentioned, such an assumption may be erroneous due to preexisting or temporary interventricular conduction abnormalities, which could present with a similar ventricular complex duration to that in the course of VT.

The aim of this analysis was determining whether human medicine algorithms for VT are applicable in veterinary medicine to differentiate wide QRS complex tachycardia in dogs.

\section{Material and Methods}

The study was performed in a retrospective fashion on a population of $11 \mathrm{dogs}$ of both sexes and various breeds and ages diagnosed with VT. The diagnosis was based on ambulatory ECG, further established based on the reaction to lidocaine or adenosine or an invasive electrophysiological study. ECG tracings were collected using a BTL-08 SD3 device with PC software (BTL, UK). The ECG tracings were next analysed using an aVR algorithm and the VT score.

In the aVR algorithm, tachycardia is differentiated based on three morphological features and the $\mathrm{Vi} / \mathrm{Vt}$ ratio (interrelationship of initial and terminal velocity of ventricular depolarisation). The morphological criteria include initiation of the QRS complex with the dominating $\mathrm{R}$ wave, the initiation of $\mathrm{QRS}$ with a $\mathrm{Q}$ or $\mathrm{R}$ wave lasting longer than $40 \mathrm{~ms}$, and finally notching of the downstroke of a predominantly negative QRS complex (QS or QRS morphology). A morphology typical for $\mathrm{VT}$ and a $\mathrm{Vi} / \mathrm{Vt}$ ratio in the aVR lead with a value $\leq 1$ is indicative of VT. Confirming one of the components of the algorithm suffices for a diagnosis of VT $(6,12)$ (Fig. 1).

The VT score is based on seven yes/no questions that include initial $\mathrm{R}$ wave in $\mathrm{V} 1$, initial $\mathrm{r}>40 \mathrm{~ms}$ in $\mathrm{V} 1 / \mathrm{V} 2$, notched $\mathrm{S}$ in $\mathrm{V} 1$, initial $\mathrm{R}$ wave in aVR, lead II $\mathrm{R}$ wave peak time $\geq 50 \mathrm{~ms}$, lack of RS complex in V1-V6, and atrioventricular dissociation. The possibility of SVT or VT is established by assessing the answers to these questions $(6,7)$.

The ECG tracings obtained from 11 dogs were reviewed using the aVR algorithm. Due to the lack of 
precordial lead tracings in three dogs, ECGs from only eight dogs were analysed using the VT score.

\section{Results}

Of the 11 tracings passed through the aVR algorithm, 10 met the VT criteria. The most common positive criterion was the $\mathrm{Vi} / \mathrm{Vt}$ ratio (8 out of 11 tracings), but it was a critical criterion for a VT diagnosis in only three tracings. The rarest morphological criterion was initiation of the QRS complex with a $\mathrm{Q}$ or $\mathrm{R}$ wave lasting longer than $40 \mathrm{~ms}$ ( 0 out of 11 ). An ECG tracing that did not indicate VT using the aVR algorithm received two points in the VT score (78\% probability).

Based on the VT score of the tracings, seven out of eight dogs had a high probability of VT:

- five tracings received two points - VT probability was $78 \%$,

- one tracing received four points - VT probability was $100 \%$,

- one tracing received three points - VT probability was $99 \%$,

- one tracing received one point - VT probability was $54.5 \%$.

The most common positive answers to questions in those diagnostic seven concerned the beginning of the QRS complex with the R wave in the V1 lead (four tracings) and a lack of $\mathrm{RS} / \mathrm{rS} / \mathrm{Rs}$ complexes in $\mathrm{V} 1-\mathrm{V} 6$ precordial leads. No positive answers were obtained with respect to the initial $\mathrm{r}>40 \mathrm{~ms}$ in the $\mathrm{V} 1$ or $\mathrm{V} 2$ leads or an interval from the beginning of the QRS to the first visible change in the direction of the initial polarity in lead II. The tracing that obtained a VT score of 0 in the aVR algorithm was classified as VT (15.5\% probability of VT).

\section{Discussion}

Heart rhythm disturbances are a common clinical problem encountered in dogs. Based on the authors' experience, their prevalence may reach $40 \%$ in animals referred to the cardiology service. Furthermore, VT was diagnosed in $4.42 \%$ of the affected population. The presence of a regular heart rate of $100 \mathrm{bpm}$ in the human (180 bpm in dogs) population may indicate VT (9).

This prevalence may increase in the population of patients with structural heart diseases (5). However, such a combination of symptoms does not suffice to make a diagnosis: a previous His bundle branch block or a block resulting from an intraventricular conduction aberrancy during tachycardia will result in similar ECG morphology during regular arrhythmia generated above the His bundle. In addition, the possibility of antidromic atrioventricular re-entrant tachycardia in a patient with an accessory pathway must be included in the differential diagnosis $(7,12)$.

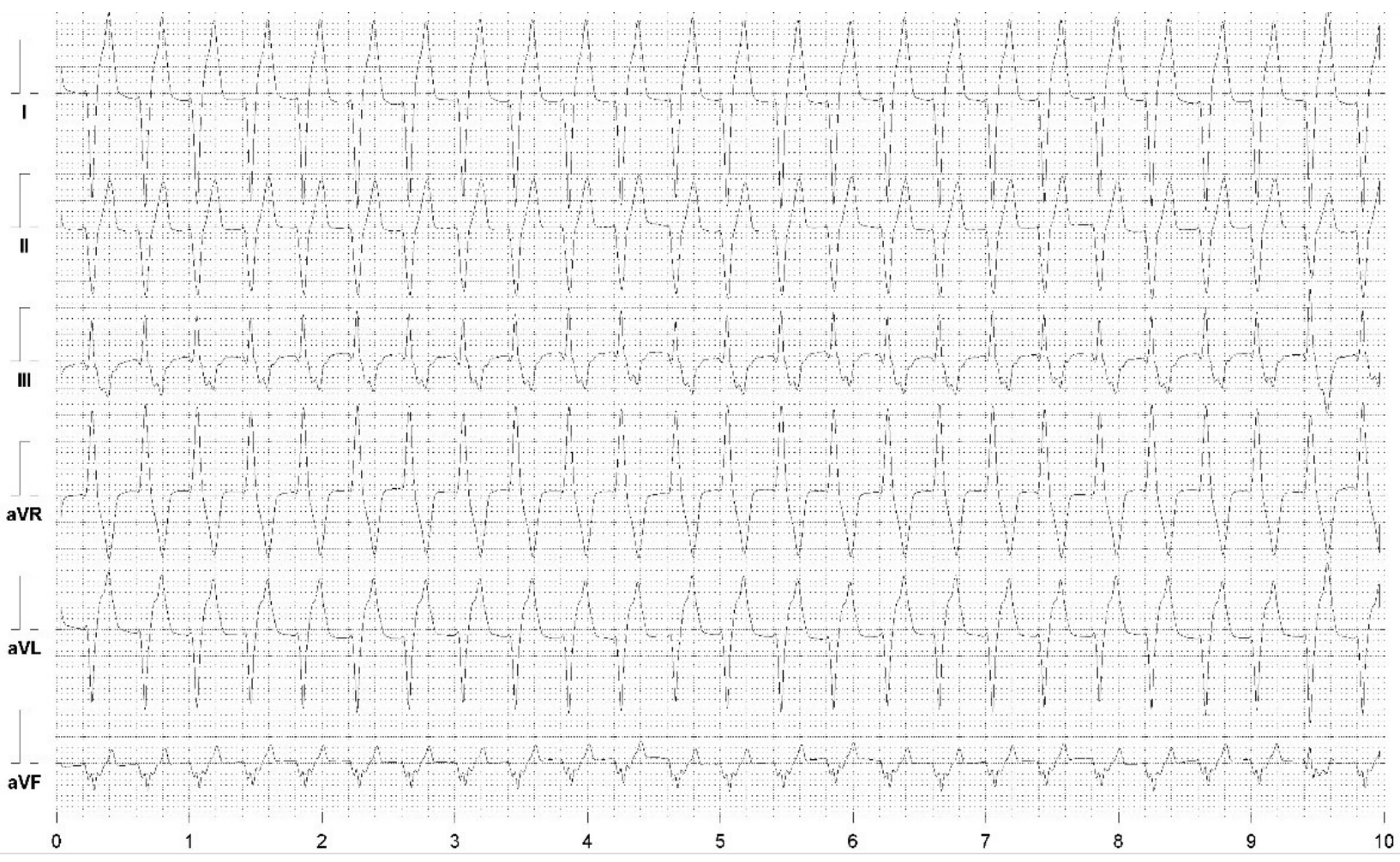

Fig. 1. Wide complex tachycardia classified as VT in the aVR algorithm. Paper speed $=25 \mathrm{~mm} / \mathrm{s} ; 10 \mathrm{~mm} / \mathrm{mV}$ 
In a patient with a leading sinus rhythm, the presence of atrioventricular dissociation with $100 \%$ specificity indicates a ventricular origin of the arrhythmia. In the case of rhythms faster than 120 150 bpm, $\mathrm{P}$ waves may not be visible within the QRS complexes, which reduces the value of the presence of an AV dissociation. In addition, careful evaluation of the repeatability of changes in the recorded curve is possible only with longer ECG recordings. Atrial fibrillation, often present in patients with cardiac disorders, and retrograde, particularly irregular (type $3: 2$, 4:3, etc.) conduction, complicate this symptom. Retrograde conduction in humans occurs in at least $20 \%$ of the population and the prevalence of this finding is unknown in the animal population (4). Moreover, the occurrence of a regular rhythm in patients with permanent atrial fibrillation proves that the rhythm originates below the atrioventricular node, and is ventricular in the vast majority of such cases. The occurrence of fusion and/or capture complexes is highly specific for ventricular arrhythmias. Such QRS morphology may exist in patients with atrial fibrillation. This finding cannot be noted in patients with efficient retrograde conduction.

In human medicine, algorithms differentiating ventricular and supraventricular tachycardia are used routinely. However, in doubtful cases, it is recommended to establish the diagnosis as wide QRS complex tachycardia and, therefore, to implement a procedure suitable for VT until definitive diagnosis from an invasive electrophysiological study is obtained (8).

The introduction of the aforementioned algorithms to veterinary medicine requires an assessment of their effectiveness on a larger group of patients, and the presented results indicate that their use may help in the diagnosis and proper treatment of VT. The interpretation of ECG often poses a practical problem for practicing veterinarians, especially in face of the different QRS morphologies. Thus, the presented algorithms may facilitate the analysis of the recordings and the diagnosis. Algorithms based only on morphological criteria may be unreliable. Apart from a typical block of the left or right bundle branch, the change of the native QRS complex may be the result of a primary or secondary pathological process. Although ischaemic injury in veterinary patients is rare, both congenital and acquired disorders as well as postinflammatory lesions may pose a diagnostic challenge. In our study carried out on a small group of dogs, we found that the sensitivity and specificity of the human algorithms used in animals was not $100 \%$, despite the animals having ventricular arrhythmia.

In veterinary practice, the aVR algorithm seems easier to use, as it requires the evaluation of only one of the ECG leads that is routinely performed in animals. As the precordial leads are not usually used in everyday veterinary practice, the Jastrzębski algorithm seems to have narrower application. However, this confirms the necessity to introduce 12 lead ECG recordings in routine clinical practice.

Based on the obtained results, the available literature data and taking into account clinical practice settings, we propose using a simplified diagnostic algorithm for wide QRS complex tachycardia in veterinary practice: the presence of AV dissociation and retrograde conduction should be treated as evidence of VT; regularity of arrhythmias with a wide QRS complex in patients with atrial fibrillation should likewise be considered evidence of VT; the morphological criteria of the aVR or VT score algorithm should be adapted to the needs of the practice, depending on the diagnostic possibilities (number of leads) of the practice and the physician's preferences; and they may be used beyond the scope of VT.

In conclusion, retrospective analysis of ECGs based on the aVR and VT score by Jastrzębski indicates that the applied algorithms may be useful in differentiating wide QRS complex tachycardia. They provide a quick, easy, and non-invasive alternative to invasive electrophysiological study, on the basis of which a precise diagnosis of the origin of tachycardia can be made. The use of the simplified diagnostic algorithm proposed by the authors may facilitate the diagnosis.

Conflict of Interests Statement: The authors declare that there is no conflict of interests regarding the publication of this article.

Financial Disclosure Statement: This research was supported by statutory funding for research and development from the Polish Ministry of Science and Higher Education assigned to the Faculty of Veterinary Medicine, Wroclaw University of Environmental and Life Sciences.

Animal Rights Statement: None required.

\section{References}

1. Brugada P., Brugada J., Mont L., Smeets J., Andries E.W.: A new approach to the differential diagnosis of a regular tachycardia with a wide QRS complex. Circulation 1991, 83, 1649-1659.

2. de Bakker J.M., van Capelle F.J., Janse M.J., Wilde A.A., Coronel R., Becker A.E., Dingemans K.P., van Hemel N.M., Hauer R.N.: Reentry as a cause of ventricular tachycardia in patients with chronic ischemic heart disease: electrophysiologic and anatomic correlation. Circulation 1988, 77, 589-606.

3. Fox P.R., Sisson D., Moise N.S.: Textbook of Canine and Feline Cardiology, W.B. Saunders, Philadelphia, 1999.

4. Gomes J.A., Dhatt M.S., Damato A.N., Akhtar M., Holder C.A.: Incidence, determinants, and significance of fixed retrograde conduction in the region of the atrioventricular node. Evidence for retrograde atrioventricular nodal bypass tracts. Am J Cardiol 1979, 44, 1089-1098.

5. Hadid C.: Sustained ventricular tachycardia in structural heart disease. Cardiol J 2015, 22, 12-24. 
6. Jastrzębski M.: A practical approach to differentiating wide QRS complex tachycardia (Praktyczne podejście do różnicowania częstoskurczów z szerokimi zespołami QRS). Przewodnik Lek 2009, 12, 20-29.

7. Jastrzębski M., Sasaki K., Kukla P., Fijorek K., Stec S., Czarnecka D.: The ventricular tachycardia score: a novel approach to electrocardiographic diagnosis of ventricular tachycardia. Europace 2016, 18, 578-584.

8. Josephson M.E.: Clinical cardiac electrophysiology. Electrophysiologic investigation: general aspects. Techniques and interpretations. Sinus node function. Lippincott Williams \& Wilkins, Philadelphia, 2002.

9. Noszczyk-Nowak A., Michałek M., Kałuża E., Cepiel A., Pasławska U.: Prevalence of arrhythmias in dogs examined between 2008 and 2014. J Vet Res 2017, 61, 103-110.
10. Sheldon S.H., Gard J.J., Asirvatham S.J.: Premature ventricular contractions and non-sustained ventricular tachycardia: association with sudden cardiac death, risk stratification, and management strategies. Indian Pacing Electrophysiol J 2010, 10, 357-371.

11. Tilley L.P., Smith F.W.K. Jr.: Electrocardiography. In: Manual of Canine and Feline Cardiology, edited by Tilley L.P., Smith Jr F.W.K., Oyama M.A., Sleeper M.M., Elsevier, St Louis, 2016, p. 56.

12. Vereckei A., Duray G., Szénási G., Altemose G.T., Miller J.M.: New algorithm using only lead aVR for differential diagnosis of wide QRS complex tachycardia. Heart Rhythm 2008, 5, 89-98. 